\title{
Jet Suppression From a Small to Intermediate to Large Radius
}

\author{
Daniel Pablos \\ Institutt for fysikk og teknologi, University of Bergen, Postboks 7803, 5020 Bergen, Norway
}

(Received 28 August 2019; revised manuscript received 2 December 2019; accepted 18 December 2019; published 3 February 2020)

\begin{abstract}
We present predictions for jet suppression from small to intermediate to very large radius, for high energy jets created in heavy ion collisions at the LHC. We find that jet suppression is surprisingly independent of the anti-kT radius $R$, first slightly increasing as one increases $R$, then at larger values of $R$ very slowly decreasing. This behavior arises from two competing effects, namely the larger energy loss of the hard jet components, which tends to increase suppression, versus the partial recovery of the lost energy due to medium response, reducing suppression.
\end{abstract}

DOI: 10.1103/PhysRevLett.124.052301

Introduction.-The strong yield suppression and substructure modification observed in the analysis of the high $p_{T}$ jets produced in heavy ion collisions compared to those measured in nucleon-nucleon collisions is ascribable to the production of deconfined QCD matter. These modifications, typically referred to as jet quenching phenomena [1], arise from the interaction of the energetic colored charges formed through parton showers with the QCD medium. The strong correlations among the thousands of low $p_{T}$ particles created in such nucleus-nucleus collisions can be very well described by hydrodynamic simulations of an exploding droplet of hot QCD liquid [2], known as the quark-gluon plasma (QGP). These simulations are surprisingly successful at describing the comparable in magnitude flowlike signals observed in smaller systems such as nucleon-nucleus and nucleon-nucleon collisions at high multiplicity [3].

One of the key aspects necessary to assess the fluid nature of the QGP consists in the determination of the measurable consequences of the response that the flowing medium would have under the passage of an energetic jet. After crossing an infinite bath of deconfined plasma, such a probe would necessarily have to become partly indistinguishable from the fluid through a process of energy degradation-it would have to hydrodynamize. The rate at which such hydrodynamization occurs depends strongly on the theoretical framework used to describe the process of energy loss, which can yield wildly different answers for the spatial distribution of the total energy of the jet after quenching. On general grounds, perturbative approaches where the coupling with the medium is required to be small

Published by the American Physical Society under the terms of the Creative Commons Attribution 4.0 International license. Further distribution of this work must maintain attribution to the author(s) and the published article's title, journal citation, and DOI. Funded by SCOAP ${ }^{3}$. typically need a rather long in-medium path in order to hydrodynamize a significative fraction of the original energy of the jet. Complementarily, nonperturbative strongly coupled computations present, as it is natural, a much faster hydrodynamization rate due to a very small mean free path. Being able to experimentally access the process of energy hydrodynamization represents in this way not only an invaluable channel with which to understand the emergence of collectivity in QCD but also allows us to confront the very different pictures of the inner workings of the QGP under which such a hydrodynamization process is described.

In this work we study the observable effects of energy and momentum hydrodynamization in the phenomenological description of jet suppression in heavy ion collisions. Jets are extended objects consisting of a collection of hadrons that are clustered together after choosing a specific reconstruction algorithm with a given jet radius parameter $R$. The larger the radius $R$, the more extended in $(\eta, \phi)$ space the jet can be, which in particular means that the jet is wider and will typically be the result of the fragmentation of a larger number of partons. Because of momentum conservation, the energy that those partons deposit in the fluid is correlated with the jet direction, and as such is part of the jet signal that is measured in experiments after the uncorrelated background is subtracted.

Given that wide jets containing a larger number of sources of energy loss tend to lose more energy than the narrower ones, based on this consideration alone the amount of jet suppression should increase with increasing jet radius $R$. However, having a larger jet radius $R$ also means that the jet retains a larger fraction of the widely distributed energy and momentum deposited in the plasma. These competing effects yield a jet suppression that is remarkably independent of the jet radius $R$ across a vast range in jet $p_{T}$. We further show that the medium response caused by the energy deposited by the recoiling jet has 
sizeable effects on the signal measured by the trigger jet. These striking long-range correlations, which depend on the pseudorapidity separation between the dijet system, are a salient feature of the assumed hydrodynamization of a large fraction of the energy of a jet and as such represent a distinctive signature of the fluidlike behavior of the QGP.

The hybrid strong/weak coupling model.-High energy jet production and evolution has been very successfully described in vacuum through perturbative QCD, both analytically and numerically through the use of Monte Carlo event generators. Jets created in heavy ion collisions are known to experience important modifications with respect to those created in proton-proton collisions, which relate both to initial state effects due to the nuclear modification of the nucleons parton distribution functions (PDFs) as well as the more dramatic final state effects associated to the interaction of the parton shower with the QGP. Even though the initial evolution of an energetic jet is dominated by the high virtuality associated to its production, $Q^{2} \sim \mathcal{O}\left(p_{T}^{2}\right)$, the presence of the medium introduces a new scale in the problem, its temperature $T \ll Q^{2}$, which is of the order of the nonperturbative $\mathrm{QCD}$ scale $T \sim \mathcal{O}\left(\Lambda_{\mathrm{QCD}}\right)$. By assuming that this nonperturbative scale dominates the physics of the interaction between an energetic probe and the QGP, it is evident that perturbative techniques alone might not suffice to describe all the dynamics behind jet quenching phenomena.

The wide scale separation allows nevertheless for an effective description, the hybrid strong or weak coupling model used in this work [4-6]. Within such a framework we use the event generator PYTHIA [7], supplemented with the leading order nuclear modifications to the PDFs as calculated in [8], to create and evolve high $p_{T}$ parton showers, to which we assign a space-time description through a formation time argument such that each parton takes a time $\tau_{f}=2 E / Q^{2}$ to split. In between splittings, partons transfer energy and momentum to the plasma hydrodynamic modes according to a strongly coupled energy loss rate that was derived within holography for $\mathcal{N}=4$ SYM, at large $N_{c}$ and infinite coupling $[9,10]$, which reads

$$
\left.\frac{d E}{d x}\right|_{\text {strongly coupled }}=-\frac{4}{\pi} E_{\text {in }} \frac{x^{2}}{x_{\text {therm }}^{2}} \frac{1}{\sqrt{x_{\text {therm }}^{2}-x^{2}}},
$$

where $E_{\text {in }}$ is the parton's initial energy and $x_{\text {therm }}=$ $\left(E_{\mathrm{in}}^{1 / 3} / T^{4 / 3}\right) / 2 \kappa_{\mathrm{sc}}$ is the maximum length that the parton can travel within the plasma before completely hydrodynamizing, with $T$ being the local temperature of the plasma as given by event-averaged hydrodynamic profiles [11]. We fixed the only free parameter of the model $\kappa_{\mathrm{sc}}$ through a global comparison to hadron and jet data at the LHC [12]. Partons that are not completely hydrodynamized are fragmented into hadrons using the Lund string model included in PYTHIA.
It has been shown that the deposition of energy and momentum from a localized source in a strongly coupled plasma generates a hydrodynamic wake after very short timescales, of $\mathcal{O}(1 / T)$ [13]. In order to estimate the measurable effects of such modifications of the QGP we can compute how the jet induced perturbations in the energy-momentum tensor of an ideal, boost-invariant fluid translate into a modification of the final hadron distribution associated with the "particlization" process that occurs after the plasma cools down at a certain hypersurface [6]. After assuming that the induced perturbations in the four-velocity and the entropy of the fluid are small, and by noting that jets propagate through the plasma approximately at a fixed space-time rapidity, we can use the standard Cooper-Frye prescription [14] to express the distribution of the hadrons coming from the wake [6], as

$$
\begin{aligned}
E \frac{d \Delta N}{d^{3} p}= & \frac{1}{32 \pi} \frac{m_{T}}{T^{5}} \cosh \left(y-y_{j}\right) \exp \left[-\frac{m_{T}}{T} \cosh \left(y-y_{j}\right)\right] \\
& \times\left\{p_{T} \Delta P_{T} \cos \left(\phi-\phi_{j}\right)+\frac{1}{3} m_{T} \Delta M_{T} \cosh \left(y-y_{j}\right)\right\},
\end{aligned}
$$

where $T$ is the temperature at the hypersurface, $m_{T} \equiv$ $\sqrt{p_{T}^{2}+m^{2}}, y$ and $\phi$ are the transverse energy, rapidity, and azimuthal angle of the hadron, respectively, while $\phi_{j}$ and $y_{j}$ are the azimuthal angle and rapidity of the parton that deposited an amount of transverse momentum $\Delta P_{T}$ and transverse energy $\Delta M_{T}$ in the plasma. This distribution can become negative, most notably along the direction opposite to the propagation of the wake. This simply means that in such a region there is a depletion of the flowing medium with respect to the unperturbed QGP due to the boost experienced by the fluid [15]. Such depletion of the plasma, which is an inseparable part of the jet signal, can amount to energy loss, or jet suppression, due to an oversubtraction effect after the uncorrelated background is subtracted as it is typically done in experiments. A useful way to picture this effect is by imagining the shape of a wave on the surface of a fluid: the ridge of the wake would correspond to the positive contributions from Eq. (2), while the trough would be associated to the negative ones. For each of the partons that interacts with the plasma we sample this closed distribution and generate hadrons whose collective energy and momentum exactly match the energy and momentum deposited by the parton.

Jet suppression as a function of the opening angle.-The results in Fig. 1 show the yield suppression of jets created in $\mathrm{PbPb}$ collisions at $\sqrt{s}=5.02 \mathrm{ATeV}$ for the $0 \%-10 \%$ centrality class compared to those in pp collisions at the same energy, quantified as it is customary in experiments through the variable $R_{\mathrm{AA}}\left(p_{T}\right) \equiv\left(d N^{\mathrm{AA}} / d p_{T}\right) /$ $\left(d N^{\mathrm{pp}} / d p_{T}\right) /\left\langle N_{\text {coll }}\right\rangle$, which is normalized to the average number of collisions in $\mathrm{PbPb}$ for the given centrality class, 


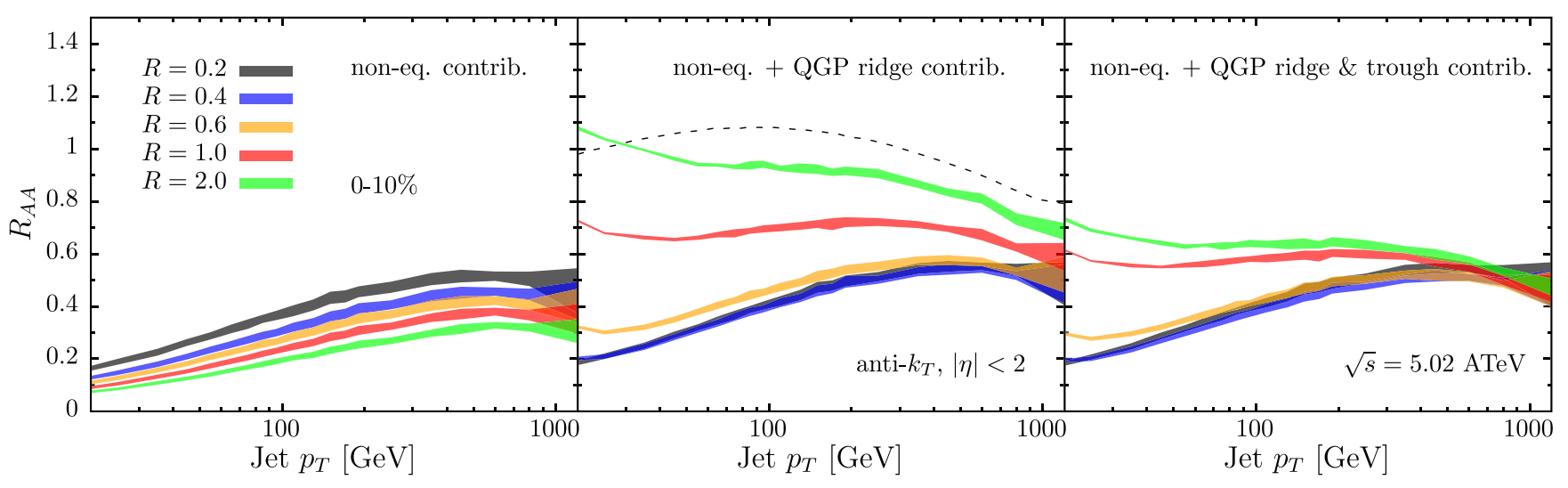

FIG. 1. The anti- $k_{T}$ jet radius $R$ dependence of $R_{\mathrm{AA}}$ in $\mathrm{PbPb}$ collisions at $\sqrt{s}=5.02 \mathrm{ATeV}$ for the $0 \%-10 \%$ centrality class. The left (middle) panel contains only the nonequilibrium (nonequilibrium + QGP ridge) contribution, while the full result that includes the effects of the QGP trough is shown in the right panel. The dashed black line in the middle panel shows the result for $R_{\mathrm{AA}}$ of $R=2$ jets without quenching.

$\left\langle N_{\text {coll }}\right\rangle$, for different values of the radius $R$ using the anti- $k_{T}$ reconstruction algorithm encoded in FastJet [17]. In order to best illustrate how the effects of the medium response affect this observable, along with the full results shown in the right panel of Fig. 1 we provide two clarifying, yet unphysical scenarios in the left and middle panels. The results shown in the left panel were obtained by taking into account only the hadrons generated through the fragmentation of the nonhydrodynamized jet partons, which we can call the nonequilibrium contribution. The visible trend of increasing suppression with increasing $R$ is due to the fact that by opening the jet cone, for a given jet $p_{T}$ one can select jets with a softer and wider fragmentation, increasing the number of energy loss sources and the total amount of energy transferred to the QGP [6,18-21].

The panel in the middle of Fig. 1 contains both the nonequilibrium particles and the excess of hadrons coming from the decay of the hydrodynamic wake generated by the passage of the jet, the QGP ridge contribution. The inclusion of these soft hadrons, spread over a wide distribution in the azimuthal angle $\phi$ with respect to the jet direction, reduces jet suppression more the larger the jet radius $R$ is. While jets with a radius of $R=0.2$ barely capture any of these hadrons, jets with $R=0.4$, in contrast to what is seen in the left panel, are now equally suppressed as those with $R=0.2$ even though the total amount of energy transferred to the plasma is larger. Given that jets typically are collimated structures, total energy loss tends to saturate as a function of the radius $R$, which means that after some intermediate value it is natural to expect that the jet retains a larger fraction of the initial hard parton momentum than compared to any smaller value of $R$. From a radius of $R=0.4$ onwards, jet suppression decreases slowly, but steadily, being necessary to go up to $R \sim 2$ to get back most of the lost energy, or equivalently to values of $R_{\mathrm{AA}} \lesssim 1$. Even though the energy is practically fully recovered within very large cones, $R_{\mathrm{AA}}$ visibly decreases at high momentum, starting from $p_{T} \gtrsim$ $300 \mathrm{GeV}$. This sizeable effect is largely due to the modification of the initial jet spectrum in $\mathrm{PbPb}$ induced by the nuclear effects on the PDFs at large values of $x$, as can be seen by the dashed black line in the middle panel of Fig. 1 where we show $R_{\mathrm{AA}}$ for $R=2$ jets without quenching. The sensitivity of jet suppression to such initial state effects is interesting per se, and can be used in the near future as a new channel with which to constrain nuclear PDFs through the standard global fit procedures.

We now discuss the right panel of Fig. 1, where all hadrons are included in the analysis. This is the actual physical situation in which the nonequilibrium contributions together with the hydrodynamized QGP ridge and trough are balanced such that the four-momentum in the event is conserved. Comparing with the middle panel of Fig. 1, where only the QGP ridge contribution was included, we can observe that jet suppression has been increased, specially for the larger values of $R$. The origin of this suppression lies in the depletion of the fluid around the jet axis induced by the passage of the recoiling jet. Whenever the cone of a given jet captures the contribution from the QGP trough induced by the fluid modification of the back-to-back jet, after subtracting what would correspond to the uncorrelated background, e.g., the background density estimated from minimum-bias events of the same centrality class where no high $p_{T}$ jet is present, the energy of the jet is reduced through an oversubtraction effect. All in all, the contribution from the nonequilibrium hadrons and those coming from the QGP ridge and trough result in a jet yield suppression that is remarkably insensitive to the radius parameter $R$ at high $p_{T}$. We predict that in order to observe a sizeable $R$ dependence one would need to go to fairly large angles, $R \gtrsim 1$, and relatively low momentum, $p_{T} \lesssim 200 \mathrm{GeV}$, a combination of values that is particularly hard to access experimentally due to the reduction of the signal to background ratio for jets with large cones at small 


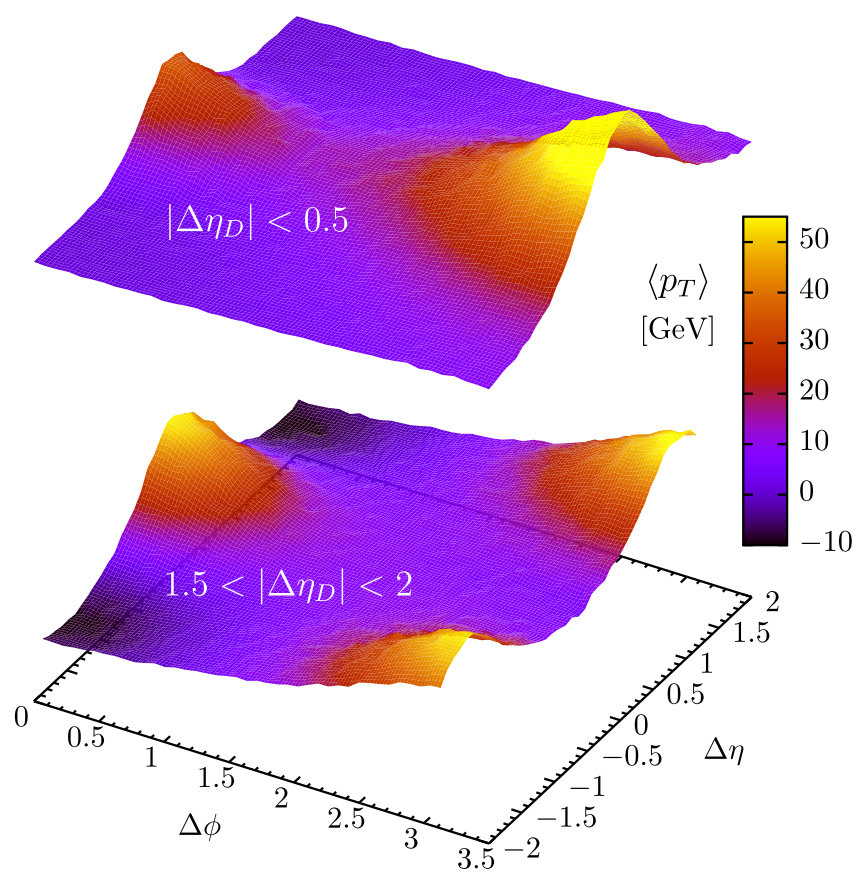

FIG. 2. The $\left\langle p_{T}\right\rangle$ density of the particles coming from the wake with respect to the leading jet direction, in terms of $\Delta \eta$ and $\Delta \phi$. The leading jet has $p_{T}^{L}>250 \mathrm{GeV}$ and the subleading has $p_{T}^{S}>80 \mathrm{GeV}$, with an angular separation in the transverse plane $\Delta \phi_{D}>2 \pi / 3$ and an absolute difference in rapidity $\left|\Delta \eta_{D}\right|$.

momentum. There is, nevertheless, a way in which the effect of the QGP trough can be quantified at relatively high jet $p_{T}$ by exploiting the lack of rapidity correlation in dijet pairs created in hadronic collisions.

The effect of the recoiling jet.-The center of mass of the hard partonic collision that produces a dijet system is in general different from the laboratory frame in which observables are measured. This obvious fact, together with the comparatively much narrower $\Delta \eta$ distribution of the soft hadrons coming from the wake with respect to their sourcing jet, as described by Eq. (2), allows us to select samples of dijets where we can engineer the amount of QGP trough contribution that the leading jet captures within its cone of radius $R$. To this end, we choose to study dijet pairs with $|\eta|<2$ where the leading jet has $p_{T}^{L}>250 \mathrm{GeV}$ and the subleading has $p_{T}^{S}>80 \mathrm{GeV}$, with an angular separation in the transverse plane $\Delta \phi_{D}>2 \pi / 3$ and an absolute difference in pseudorapidity $\left|\Delta \eta_{D}\right|$, which we vary. A very convenient way to visualize the contributions from the QGP ridge and trough in a dijet system is by plotting the $\left\langle p_{T}\right\rangle$ density of the hydrodynamized particles as a function of their separation in azimuthal angle $\Delta \phi$ and pseudorapidity $\Delta \eta$ with respect to the leading jet, as we show in Fig. 2. The top plot in Fig. 2 corresponds to dijet configurations of jets with $R=1$ that are close in pseudorapidity, namely $\left|\Delta \eta_{D}\right|<0.5$, while the dijet samples from the bottom plot have a larger separation of $1.5<\left|\Delta \eta_{D}\right|<2$. In the top plot we can clearly see the
QGP ridge contribution both at the near side, with $\Delta \phi \sim 0$ and at the away side, with $\Delta \phi \sim \pi$. The size of the QGP ridge is greater in the away side because the subleading jet has on average lost more energy to the plasma than the leading jet at the near side due to its lower jet $p_{T}$ cut. The negative $\left\langle p_{T}\right\rangle$ density contribution associated to the QGP trough generated by the subleading jet cannot be seen with clarity in the top plot because it falls on top the QGP ridge contribution generated by the wake of the leading jet. When the dijet system is well separated in pseudorapidity, as presented in the lower plot of Fig. 2, we can see a black dip of depleted fluid at the near side, centered around the pseudorapidity at which the subleading jet sits with respect to the leading jet. The fact that there is barely any signal of the QGP trough in the away side basically reflects that the leading jet has on average lost comparatively much less energy than the subleading jet. What this means in terms of jet energy loss is that in the situations in which the QGP trough generated by the subleading jet does not hit the area defined by the leading jet cone of radius $R$, the latter experiences less suppression and its $R_{\mathrm{AA}}$ is increased compared to the case in which the pseudorapidity separation is small.

This dependence on jet suppression as a function of the pseudorapidity separation between a dijet system can be easily quantified by computing the leading jet yield suppression, which we can call $R_{\mathrm{AA}}^{\text {lead }}$, as a function of the $\left|\Delta \eta_{D}\right|$ between the leading and subleading jets. In Fig. 3 we present such computation for intermediate jet radius with $R=0.4$ in the top panel and large jet radius $R=1$ in the bottom panel. Similarly to Fig. 1, we present three sets of results corresponding to the nonequilibrium contribution only, in black, the nonequilibrium plus the QGP ridge, in blue, and all the contributions, including the QGP trough, in red. The first thing we observe is the approximate flatness of leading jet suppression for a wide range of $\left|\Delta \eta_{D}\right|$ for the two sets of results that omit the contribution from the QGP trough, both for $R=0.4$ and $R=1$ jets. The reason for the drop of $R_{A A}^{\text {lead }}$ at the highest values of $\left|\Delta \eta_{D}\right|$ is because of the hard parton $p_{T}$ spectrum becoming steeper due to the requirement that both the leading and subleading jets sit at relatively large pseudorapidities. The dependence on $\left|\Delta \eta_{D}\right|$ comes when we include the effect of the QGP trough generated by the wake of the more quenched subleading jet. As expected from simple geometrical considerations, the effect of the recoiling jet is gradually reduced as one increases $\left|\Delta \eta_{D}\right|$, being almost irrelevant beyond $\left|\Delta \eta_{D}\right| \gtrsim R+\sigma(\Delta \eta)$, where $\sigma(\Delta \eta) \simeq 1$ symbolizes the range in pseudorapidity below which most of the particles from the wake sit, as described by Eq. (2). Interestingly, given that most of the hydrodynamized energy is sourced from the center of the collimated jets, the rate at which $R_{A A}^{\text {lead }}$ increases with $\left|\Delta \eta_{D}\right|$ presents a knee around $\left|\Delta \eta_{D}\right| \sim R$, as can be more clearly appreciated in the bottom panel of Fig. 3 . 


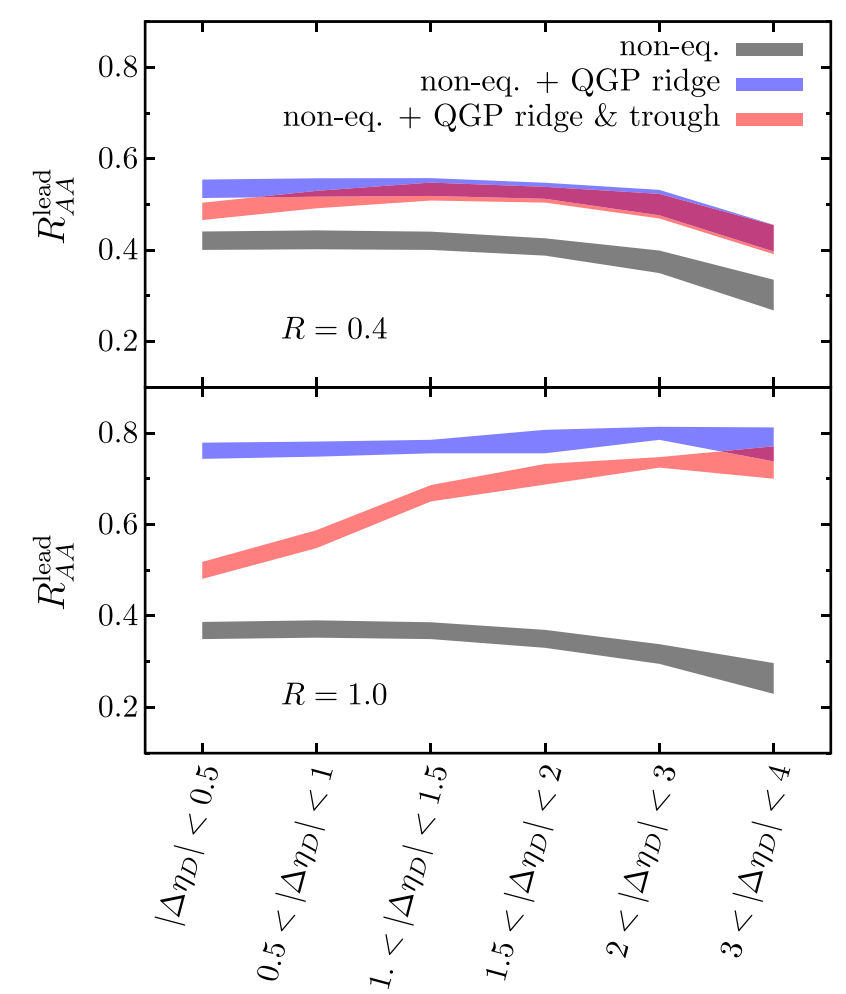

FIG. 3. Jet suppression $R_{\mathrm{AA}}^{\text {lead }}$ for leading jets above $p_{T}^{L}>250 \mathrm{GeV}$, as a function of the pseudorapidity separation $\left|\Delta \eta_{D}\right|$ with respect to the back-to-back subleading jet with $p_{T}^{S}>80 \mathrm{GeV}$. The top panel is for jets with an anti- $k_{T}$ radius $R=0.4$, while the lower panel is for jets with $R=1$. Here, "noneq." refers to the contribution coming from the nonhydrodynamized fraction of the jet energy.

Conclusions. - The angular dependence of jet suppression encodes key information about the process of energy and momentum hydrodynamization, and for this reason can be used to greatly improve our understanding of fundamental aspects of the jet/QGP interaction. Distinctive features of the efficient hydrodynamization process that follow our assumption of energy loss at strong coupling are seen through the remarkable independence of inclusive jet suppression from the jet opening angle $R$, which in particular means that $R_{\mathrm{AA}}$ does not go to 1 even for very large radius, contrary to current expectations. We have shown that this fact does not contradict energy and momentum conservation and that it is a consequence of the effect of the trough associated to the wake generated by the recoiling jet. The dependence of leading jet suppression on the pseudorapidity separation of a dijet system allows us to present a set of predictions for the discovery of the effect of the QGP trough and, by extension, one of the most significant tests from jet quenching dynamics of the fluidlike behavior of the QGP.

We thank Carlota Andrés, Jasmine Brewer, Jorge Casalderrey-Solana, Yi-Lun Du, Yen-Jie Lee, Chris
McGinn, Hannu Paukkunen, Krishna Rajagopal, Ádám Takács, and Konrad Tywoniuk for useful discussions. This work is supported by a grant from the Trond Mohn Foundation (Grant No. BFS2018REK01).

Note added.-Recently, new data from CMS [22] and ALICE [23] have been released, showing that there is a marked independence of jet suppression with the jet radius $R$, in qualitative agreement with our predictions.

[1] Y. Mehtar-Tani, J. G. Milhano, and K. Tywoniuk, Int. J. Mod. Phys. A 28, 1340013 (2013).

[2] S. Acharya et al. (ALICE Collaboration), J. High Energy Phys. 09 (2018) 006.

[3] R. D. Weller and P. Romatschke, Phys. Lett. B 774, 351 (2017).

[4] J. Casalderrey-Solana, D. C. Gulhan, J. G. Milhano, D. Pablos, and K. Rajagopal, J. High Energy Phys. 10 (2014) 019; 09 (2015) 175.

[5] J. Casalderrey-Solana, D. C. Gulhan, J. G. Milhano, D. Pablos, and K. Rajagopal, J. High Energy Phys. 03 (2016) 053.

[6] J. Casalderrey-Solana, D. Gulhan, G. Milhano, D. Pablos, and K. Rajagopal, J. High Energy Phys. 03 (2017) 135.

[7] T. Sjöstrand, S. Ask, J. R. Christiansen, R. Corke, N. Desai, P. Ilten, S. Mrenna, S. Prestel, C. O. Rasmussen, and P.Z. Skands, Comput. Phys. Commun. 191, 159 (2015).

[8] K. J. Eskola, H. Paukkunen, and C. A. Salgado, J. High Energy Phys. 04 (2009) 065.

[9] P. M. Chesler and K. Rajagopal, Phys. Rev. D 90, 025033 (2014).

[10] P. M. Chesler and K. Rajagopal, J. High Energy Phys. 05 (2016) 098.

[11] C. Shen, Z. Qiu, H. Song, J. Bernhard, S. Bass, and U. Heinz, Comput. Phys. Commun. 199, 61 (2016).

[12] J. Casalderrey-Solana, Z. Hulcher, G. Milhano, D. Pablos, and K. Rajagopal, Phys. Rev. C 99, 051901(R) (2019).

[13] P. M. Chesler and L. G. Yaffe, Phys. Rev. Lett. 99, 152001 (2007).

[14] F. Cooper and G. Frye, Phys. Rev. D 10, 186 (1974).

[15] This depletion effect has also been reported in analysis where the deposited energy is treated as a source term in the hydrodynamic evolution equations [16].

[16] W. Chen, S. Cao, T. Luo, L. G. Pang, and X. N. Wang, Phys. Lett. B 777, 86 (2018).

[17] M. Cacciari, G. P. Salam, and G. Soyez, Eur. Phys. J. C 72, 1896 (2012).

[18] J. G. Milhano and K. C. Zapp, Eur. Phys. J. C 76, 288 (2016).

[19] K. Rajagopal, A. V. Sadofyev, and W. van der Schee, Phys. Rev. Lett. 116, 211603 (2016).

[20] J. Brewer, K. Rajagopal, A. Sadofyev, and W. van der Schee, J. High Energy Phys. 02 (2018) 015.

[21] J. Casalderrey-Solana, G. Milhano, D. Pablos, and K. Rajagopal, arXiv:1907.11248.

[22] CMS Collaboration, Report No. CMS-PAS-HIN-18-014.

[23] R. Haake (ALICE Collaboration), arXiv:1909.01639. 